\title{
Device Fabrication, Characterization, and Thermal Neutron Detection Response of LiZnP and LiZnAs Semiconductor Devices
}

Benjamin W. Montag ${ }^{1}$, Philip B. Ugorowski ${ }^{1}$, Kyle A. Nelson ${ }^{1}$, Nathaniel S. Edwards ${ }^{1}$, Douglas S. McGregor ${ }^{1}$

${ }^{1}$ Semiconductor Materials and Radiological Technologies (S.M.A.R.T) Laboratory, Kansas State University, Manhattan, KS 66506, U.S.A.

\section{Corresponding Author Contact:}

Benjamin W. Montag

Email: bmontag@ksu.edu

Tel: 785:532:6480

Fax: 785:532:7057

Keywords: Lithium compounds; Radiation; Neutron Detector; Semiconducting ternary compounds

\begin{abstract}
Nowotny-Juza compounds continue to be explored as candidates for solid-state neutron detectors. Such a device would have greater efficiency, in a compact form, than present day gasfilled ${ }^{3} \mathrm{He}$ and ${ }^{10} \mathrm{BF}_{3}$ detectors. The ${ }^{6} \mathrm{Li}(\mathrm{n}, \mathrm{t}){ }^{4} \mathrm{He}$ reaction yields a total Q-value of $4.78 \mathrm{MeV}$, larger than ${ }^{10} \mathrm{~B}$, an energy easily identified above background radiations. Hence, devices fabricated from semiconductor compounds having either natural $\mathrm{Li}$ (nominally $7.5 \%{ }^{6} \mathrm{Li}$ ) or enriched ${ }^{6} \mathrm{Li}$ (usually $\left.95 \%{ }^{6} \mathrm{Li}\right)$ as constituent atoms may provide a material for compact high efficiency neutron detectors. Starting material was synthesized by preparing equimolar portions of $\mathrm{Li}, \mathrm{Zn}$, and As sealed under vacuum ( $10^{-6}$ Torr) in quartz ampoules lined with boron nitride and subsequently reacted in a compounding furnace [1]. The raw synthesized material indicated the presence high impurity levels (material and electrical property characterizations). A static vacuum sublimation in quartz was performed to help purify the synthesized material [2,3]. Bulk crystalline samples were grown from the purified material $[4,5]$. Samples were cut using a diamond wire saw, and processed into devices. Bulk resistivity was determined from I-V curve measurements, ranging from $10^{6}-10^{11} \Omega \mathrm{cm}$. Devices were characterized for sensitivity to $5.48 \mathrm{MeV}$ alpha particles, $337 \mathrm{~nm}$ laser light, and neutron sensitivity in a thermal neutron diffracted beam at the Kansas State University TRIGA Mark II nuclear reactor. Thermal neutron reaction product charge induction was measured with a LiZnP device, and the reaction product spectral response was observed.
\end{abstract}




\section{INTRODUCTION}

Nowotny-Juza compounds were originally, and are still today, studied for photonic applications [6-8]. The filled tetrahedral compound class $\mathrm{A}^{\mathrm{I}} \mathrm{B}^{\mathrm{II}} \mathrm{C}^{\mathrm{V}}$ materials consist of the III-Vlike compounds with lithium interstitials. These materials are desirable for their zincblende cubic crystal structure, and unlike thin-film coated and Li-doped devices, the concentration of Li atoms is equal to other constituent atoms. The zincblende crystal structure is typically arranged with a F$43 \mathrm{~m}$ space group, where the group II atom is located at $\tau_{1}=(0,0,0) a$ where $a$ is the lattice constant, and the group $\mathrm{V}$ atom is located at $\tau_{2}=(1 / 4,1 / 4,1 / 4) a$. The spacious cubic structure allows for lithium atoms to fill the interstitial site at $\tau_{3}=(1 / 2,1 / 2,1 / 2) a$. The filling of these interstitial sites allows for a lithium-loaded semiconducting material.

Materials containing ${ }^{6} \mathrm{Li},{ }^{10} \mathrm{~B},{ }^{113} \mathrm{Cd},{ }^{157} \mathrm{Gd}$ and ${ }^{199} \mathrm{Hg}$ have been considered for solid-state neutron detectors [9-20]. The ${ }^{10} \mathrm{~B}(\mathrm{n}, \alpha)^{7} \mathrm{Li}$ reaction is desirable for the ${ }^{10} \mathrm{~B}$ microscopic thermal neutron absorption cross section of 3839 barns, but boron-based compounds, such as BP, BN, and BAs have shown limited success, and thus far do not appear promising due to crystal growth and materials preparation problems [17-20]. Thin-film boron devices suffer due to their geometry, where only one reaction product can be absorbed in semiconducting material, therefore producing some signals that can be difficult to distinguish from background and gamma-ray induced events [21]. Additionally, because of the reaction product self-absorption, the reactive film thickness is limited, and therefore thermal neutron absorption is limited, consequently resulting in a maximum intrinsic detection efficiency of approximately $4.5 \%$ [21]. Solid-state detectors containing ${ }^{113} \mathrm{Cd}$ and ${ }^{199} \mathrm{Hg}$ devices also have limited detection efficiency due to the low absorption probability of the prompt gamma-rays that result from the ${ }^{113} \mathrm{Cd}(\mathrm{n}, \gamma){ }^{114} \mathrm{Cd}$ and ${ }^{199} \mathrm{Hg}(\mathrm{n}, \gamma){ }^{200} \mathrm{Hg}$ reactions [9-12]. The ${ }^{157} \mathrm{Gd}(\mathrm{n}, \gamma){ }^{158} \mathrm{Gd}$ reaction is desirable for the large ${ }^{157} \mathrm{Gd}$ thermal neutron capture cross section of 240,000 barns. Unfortunately, the ${ }^{157} \mathrm{Gd}(\mathrm{n}, \gamma){ }^{158} \mathrm{Gd}$ reaction yields a spectrum of low energy prompt gamma rays and, low energy conversion electrons, all of which are difficult to discern from background radiations [21]. Finally, ${ }^{6} \mathrm{Li}$-based semiconductors have not been explored to the same extent as other thermal neutron absorbers, and has an intrinsic thermal neutron absorption cross section of 940 barns. The reaction produces a total Q-value of $4.78 \mathrm{MeV}$, described by the following reaction, 


$$
{ }^{6} \mathrm{Li}+{ }_{0}^{1} n \rightarrow{ }^{3} \mathrm{H}(2.73 \mathrm{MeV})+\alpha(2.05 \mathrm{MeV}) .
$$

LiZnP was studied in the past as a possible radiation detector material [22], however there is no evidence of a published document from that particular study. In this study, the ternary products, LiZnP and LiZnAs were synthesized, purified and grown into bulk crystalline ingots [15]. Described in the following study is a procedure for device fabrication from LiZnP and LiZnAs materials, and details of the subsequent device characterization. I-V curves were collected and a bulk resistivity was determined for each sample. Samples were characterized for sensitivity to alpha particle radiation and $337 \mathrm{~nm}$ laser light. Upon demonstrating that charge carriers could be transported through the material, samples were exposed to thermal neutrons, and thermal neutron reaction product spectra were collected.

\section{EXPERIMENTAL PROCEDURES}

Described in the following work is a method developed for material processing and device fabrication of the hygroscopic LiZnP and LiZnAs materials. Also described are the methods used to characterize the devices.

\section{Device processing and fabrication}

LiZnP and LiZnAs were synthesized, as described elsewhere, in small batches up to 2.0 grams and 6.0 grams, respectively [1]. The synthesized material was purified by a static sublimation process. The process appeared to successfully separate both the ternary LiZnP and LiZnAs material, from elemental and binary residual materials that often result from the synthesis process $[2,3]$. This purified LiZnP and LiZnAs material were subsequently grown into bulk crystals under a high-temperature vertical Bridgman technique [4, 5].

LiZnP and LiZnAs samples of various sizes were cut from bulk ingots using a Laser Technology West Ltd. CS400 diamond wire saw while under a constant flow of mineral oil for protection from moisture in the air. LiZnP and LiZnAs samples were diced into various sizes up to $2.10 \times 4.06 \times 4.16 \mathrm{~mm}^{3}$. Samples were prepared for polishing by mounting to a polishing fixture 
by paraffin wax. The sample face was polished with a series of silicon carbide polishing papers ranging from 15.3 down to 2.5 micron grain size, ANSI grit: 600 (P1200), 800 (P2400), and 1200 (P4000) [23, 24]. A final polish was performed on all sample sides with a mineral oil slurry over 4000 grit $\mathrm{SiC}$ paper. The surface of the sample was wiped clean with a hexane saturated cotton swab. Samples were placed into a shadow mask for contact patterning and the loaded shadow mask was placed into an air-tight container so as to transport it from an argon glove box. This containment prevented moisture exposure to the samples while in transition. The shadow mask was subsequently, rapidly, loaded onto the planetary of the electron-beam evaporator, the door shut, followed by immediate evacuation of the chamber. Metal contacts were evaporated onto opposing polished surfaces of the ternary material; $500 \AA$ of titanium followed by $8,000-10,000$ $\AA$ A of gold. Once complete, the electron beam chamber was back-filled with nitrogen, opened, and the shadow mask (containing the samples) was removed quickly, placed into an argon glove box anti-chamber and evacuated. Numerous LiZnP and LiZnAs samples were processed and fabricated into devices with this method, as shown in Fig. 1.

\section{Device Characterization}

To confirm the conductivity/resistivity of the sample, I-V curves were collected with a Keithley 237 High Voltage Source Measurement Unit coupled with a LabView interface on all processed samples prior to other measurements. Devices showing ohmic or Schottky behavior were tested for sensitivity to $5.48 \mathrm{MeV}$ alpha particles. The sides of the device were coated with HumiSeal ${ }^{\circledR} 1 \mathrm{~B} 73$ to protect the materials from moisture in the air. An ${ }^{241} \mathrm{Am}$ spectrum was collected initially with a CZT sample to insure that the measurement setup was operational. Once verified, the $\mathrm{LiZnP}$ or LiZnAs sample was mounted into a project box with adjustable pressure contacts that connected firmly to each contact surface, as shown in Fig. 3. The project box with a mounted sample was connected to a charge-sensitive preamplifier (Ortec 142A), and the preamplifier signal was connected to a Canberra 2022 amplifier, and the output amplifier signal was connected to an Ortec TRUMP PCI multichannel analyzer. The high voltage was applied with a Matsusada Precision Inc power supply. An ${ }^{241} \mathrm{Am}$ alpha particle source (conductive source housing) was placed between the ground and the cathode of a sample where the alpha particle 
induced signal would be primarily made up of electrons. Pulse height spectra were collected with this configuration.

Devices were also tested for sensitivity to $337 \mathrm{~nm}$ laser light. $337 \mathrm{~nm}$ light is in the blue ultraviolet range and has an energy of $3.68 \mathrm{eV}$, high enough energy to excite electron - hole pairs in LiZnAs and LiZnP (reported bandgap of $1.51 \mathrm{eV}$ for LiZnAs [25] and $2.04 \mathrm{eV}$ for LiZnP [26]). Devices were coated in Epoxy Technology 301-2 conformal coating, a coating that was verified experimentally to be transparent to 337 photons. The experimental setup included a Laser Photonics LN300 laser with an energy of $250 \mu \mathrm{J}$ per pulse at $337 \mathrm{~nm}$. The pulse width was $5 \mathrm{~ns}$ with beam dimensions of 9 x $4 \mathrm{~mm}$ (hor x ver). A LiZnAs sample of dimensions 1.43 x $3.96 \mathrm{x}$ $2.45 \mathrm{~mm}^{3}$ was mounted in a project box that allowed for light to pass through the back of the project box so light could be absorbed in a Si witness photodetector. The experimental arrangement included an adjustable iris, silicon photodetector and an Agilent Infiniium 54832D MSO oscilloscope as shown in Fig. 2. The iris was adjusted to reduce the beam to approximately 1.0 $\mathrm{mm}$ diameter incident on the $3.96 \times 2.45 \mathrm{~mm}$ face of the sample. The intensity, or response, from the Si photodiode was recorded on an oscilloscope with and without the sample in the incident beam to confirm $337 \mathrm{~nm}$ light was being absorbed. Afterwards, the $337 \mathrm{~nm}$ laser pulse was traced using the $1.43 \times 3.96 \times 2.45 \mathrm{~mm}^{3}$ LiZnAs sample. The setup consisted of the same collimating iris open to approximately $1.0 \mathrm{~mm}$, along with an Agilent Infiniium 54832D MSO oscilloscope, and a Melles Griot wide bandwidth amplifier (power supply, preamp and trans-impedance amplifier package). The wide bandwidth amplifier was a current amplifier, used generally for operating photodiodes where the high gain allows for accurate detection of photocurrents from as low as $100 \mathrm{pA}$ up to $2 \mathrm{~mA}$. A 10.0 volt bias was applied to the sample (at the device) and the laser was at $1 \mathrm{~Hz}$ pulse frequency. The resulting pulse was observed on the oscilloscope. Additional LiZnAs and LiZnP samples were tested with the same configuration.

Neutron sensitivity tests were performed at the Kansas State University TRIGA Mark II nuclear reactor. A thermalized neutron beam from a diffractometer beam port was used for the majority of the testing. A beam of $110 \pm 10$ neutrons $\mathrm{cm}^{-2} \mathrm{sec}^{-1} \mathrm{~kW}^{-1}$, calibrated with a ${ }^{3} \mathrm{He}$ detector, is present at beam port test location. The radial beam from the reactor core is diffracted with a pyrolytic graphite crystal oriented to diffract thermal neutrons $(0.0259 \mathrm{eV})$ from the primary collimated neutron beam into the sample test area. All detector testing presented here was performed under vacuum ( $<50 \mathrm{mTorr}$ ) in a Canberra vacuum chamber, designed for a surface 
barrier alpha particle detector, to prevent free air ionization from occurring. Devices were evaluated for thermal neutron sensitivity at a variety of applied bias through an Ortec 142A preamplifier which was connected to a Canberra 2022 amplifier, where the output was connected to an Ortec PCI TRUMP multichannel analyzer. Thermal neutron reaction product spectra were collected.

\section{RESULTS AND CONCLUSIONS}

\section{I. $\quad$ I-V curves}

The LiZnP and LiZnAs devices generally had relatively high conductivity at $1-2$ volt forward and reverse bias. The leakage current would reach compliance of the curve tracing instrument at $100 \mu$ volts, rendering the device inoperable due to the high conductivity. The high conductivity was observed more frequently with devices fabricated with silver epoxy contacts. Material purification post ternary synthesis [2,3] reduced the conductivity, and employing Ti/Au contacts also helped produce relatively stable devices, where silver epoxy contacts always resulted in high conductivity devices. Many devices fabricated from bulk grown ingots processed from the in-house synthesized and purified material exhibited a typical ohmic resistance I-V curve, as shown in Fig. 4. Some devices, however, displayed rectifying characteristics as shown in Fig. 5. The rectifying behavior assisted with noise reduction [27] and allowed the device to operate at a voltages $<10$ volts. It was observed that excessive bias and/or time at a selected bias would eventually cause the device to breakdown, resulting in high conductivity and electronic noise.

\section{Alpha particle and 337nm laser testing}

Purified samples of LiZnP and LiZnAs were tested for alpha particle sensitivity. TRIM SRIM [28] simulations indicated that a $5.48 \mathrm{MeV}$ alpha particles are blocked by a contact composed of a $500 \AA$ Ti layer followed by 94,300 $\AA$ Au layer. However, only 10,000 $\AA$ of Au were deposited, indicating that $5.48 \mathrm{MeV}$ alpha particles will pass through the contact and enter the LiZnP material. It was observed that there was an optimal voltage where the output signal was 
strongest. Shown in Fig. 6 is a ${ }^{241}$ Am spectrum with an applied bias of 50 volts. At higher applied voltages, the signal would often become either extremely noisy, or signals would be lost.

Purified samples of LiZnAs were tested for sensitivity to $337 \mathrm{~nm}$ laser light. The initial study confirmed that light was absorbed in the LiZnAs sample. The intensity, or response, from the Si photodiode was recoded on the oscilloscope without the sample present as shown in Fig. 7 (top), and with the LiZnAs sample in the beam (bottom). By comparing the pulse intensities, where the initial unattenuated intensity, $I_{o}$, was $500 \mathrm{mV}$, and the attenuated intensity, $I(1.43 \mathrm{~mm})$, was 95 $\mathrm{mV}$, it was determined that approximately $81 \%$ of the photons were absorbed by $1.43 \mathrm{~mm}$ of LiZnAs (assuming negligible absorption in the conformal coating). The surface of an equivalent LiZnAs sample from the same growth ampoule was polished using 4000 grit polishing paper and tested for absorption with the same aforementioned experimental setup and resulted in approximately $81 \%$ absorption, indicating minimal surface reflection. Using these parameters an absorption coefficient was determined with

$$
I(t)=I_{o} e^{-x t},
$$

where $t$ is thickness of the absorber, $x$ is the absorption coefficient, $I(t)$ is the intensity at thickness $t$, and $I_{o}$ is the initial intensity. An absorption coefficient of $0.147 \mathrm{~mm}^{-1}$ was determined. The absorption coefficient was used to determine an average thickness of absorption

$$
<t>=\frac{\int_{0}^{t} t I_{o} e^{-x t} d t}{\int_{0}^{t} I_{o} e^{-x t} d t},
$$

where $t$ is the sample thickness and $x$ is the absorption coefficient. Eq. (3) was integrated from 0 to $1.43 \mathrm{~mm}$, the sample thickness, and an average thickness for absorption was found to be 0.69 $\mathrm{mm}$. This result was a used to confirm that $337 \mathrm{~nm}$ laser light was being absorbed in LiZnAs.

Laser pulses of $337 \mathrm{~nm}$ photons were traced with LiZnAs samples. The LiZnAs sample of dimensions $1.59 \times 1.39 \times 1.42 \mathrm{~mm}^{3}$ was placed between the contacts of a project box. A 10.0 volt bias was applied to the sample (at the device) and the laser was set to pulse at approximately $1 \mathrm{~Hz}$. A resulting pulse collected on the oscilloscope is shown in Fig. 8. Approximately a $5 \mathrm{mV}$ pulse 
was recorded with the LiZnAs sample. Other samples were tested which included LiZnAs sample of dimensions $1.43 \times 3.96 \times 2.45 \mathrm{~mm}^{3}$. This particular sample was much more sensitive to the 337 $\mathrm{nm}$ photons than the previous sample, and resulted in about a $30 \mathrm{mV}$ pulse height as shown in Fig. 9. These studies further indicate that charge carriers can be transported through LiZnP and LiZnAs devices. Additionally, the low noise that was observed in these studies showed how important purification of the synthesized material is to the device electrical properties. The relatively high noise and noise bursts that were commonly observed in unpurified LiZnP and LiZnAs devices were not observed in the initial testing of devices fabricated from purified LiZnP and LiZnAs materials.

\section{Neutron Sensitivity}

Neutron testing proved challenging for two fundamental reasons, (1) alignment of the small detectors in the neutron beam and (2) intermittent noise problems. Unpurified samples would unfortunately suffer from noise "bursts" that would disrupt the measurement. Shielding and common grounding were added to the experimental arrangement to eliminate possible outside noise contribution from ground loops and mechanical equipment in the surrounding area. However, these precautions did not eliminate the noise bursts. It is suspected that a large charge trapping and a long delayed charge de-trapping mechanism may likely be occurring in the devices, which required many measurements to be discarded after a noise burst event. To reduce the probability of experiencing a noise burst, short measurement collection times were used, typically in the range of $3-5$ min. Additionally, LiZnP and LiZnAs would often break down from high biases applied over a long period of time. Generally, no more than \pm 60 volts at the detector was applied during testing. Noise bursts were observed to cause irreparable damage to the device, resulting in a constant noisy device at any voltage applied in subsequent testing. I-V characteristic data was collected before radiation sensitivity testing. It was observed that the devices became conductive after neutron radiation testing (where a high voltage was applied to the device for a long period of time). Devices were reprocessed, where electrical contacts were applied to a different crystalline direction, which, in some cases, resurrected the device for further testing.

Neutron sensitivity was evaluated for the bulk crystalline unpurified LiZnP and LiZnAs devices that had silver epoxy anode and cathode contacts, and also for devices with a Ti-Au anode 
and silver epoxy cathode contacts. The Ti-Au anode contact appeared to help reduce the noise to a manageable level and allow for testing; however, the aforementioned noise bursts were continued to be a problem, and appeared more frequently as testing continued. These noise bursts required testing to be continuously monitored. An example of one the best of the spectra collected is shown in Fig. 10 for spectra collected with a cadmium shutter open (neutrons) and closed (no neutrons). An applied bias of -10 volts was applied to the detector. The 300-second measurement was continuously monitored where a noise burst did not distort the signal. It was concerning that a noise edge was not observed in the shutter closed measurement. It would be expected that the noise edge would also be present with the shutter closed. Nevertheless, the noise bursts and neutron sensitivity testing indicated that there was an obvious material problem that needed to be addressed, and was likely a result of material impurities. Hence, static sublimation purification was performed for the synthesized material and bulk ingots were grown from the purified material.

Devices were fabricated, and tested from the purified material. The noise bursts observed with unpurified samples were not observed with purified devices. However, the breakdown in device performance after prolonged testing continued to be a problem. Additionally, initial tests with purified samples showed that some devices performed better than others. For example, the thermal neutron response pulse height spectrum from a LiZnP sample indicated neutron sensitivity for a 300 second measurement, as shown in Fig. 11. Desirable features were observed in the pulse height spectrum, yet, these measurements were not always repeatable. The applied bias was also increased to observe the effect on performance. Generally, at this point in the experimental process, the device became noisy and resulted in spectral responses as shown in Fig. 12. Spectral features disappeared and the noise amplitude increased, appearing above channel 100. The only differentiating feature between the "shutter open" and "shutter closed" measurements was the slightly higher noise spectrum that extended into the higher energy spectrum with the presence of neutrons. Other LiZnP samples were tested, resulting in no observable response to thermal neutrons, or only an extension of the noise spectrum to higher channel numbers, but not resulting in any distinguishable pulse height features. Noise eventually became so prominent in the samples that spectra would become saturated with noise within all channels. Afterwards, I-V curves were collected, and high conductivity was observed in all cases.

Purified LiZnAs samples were also tested for neutron sensitivity at the thermal neutron beam port of the KSU TRIGA Mark II nuclear reactor. Similar to what was observed with LiZnP 
samples, the measured LiZnAs sensitivity to neutrons was minimal. A pulse height spectrum was collected for 600 seconds for each sample, an example shown in Fig. 13. For each measurement, the reactor power was at $250 \mathrm{~kW}$ and the bias 53 volts applied through the preamplifier. The applied bias was increased to determine an optimal bias, but instead introduced increased noise problems.

\section{DISCUSSION}

Devices were fabricated from in-house synthesized and purified LiZnP and LiZnAs materials. Devices fabricated from unpurified material with silver epoxy contacts were often highly conductive, rendering the devices inoperable. The purification process, along with evaporated $\mathrm{Ti} / \mathrm{Au}$ contacts, produced much higher resistivity and reliable devices. Measured resistivities were often high for the initial tests $\left(10^{6}-10^{11} \Omega \mathrm{cm}\right)$. Devices were tested for sensitivity to $337 \mathrm{~nm}$ laser light. It was determined experimentally that for a LiZnAs device of $1.43 \mathrm{~mm}$ thickness, approximately $81 \%$ of the incident photons from the $250 \mu \mathrm{J}$ laser pulse were absorbed in the material with minimal losses to reflection. An absorption coefficient was determined to be $0.147 \mathrm{~mm}^{-1}$ for $337 \mathrm{~nm}$ photons. A pulse height of approximately $30 \mathrm{mV}$ and a pulse width of approximately $70 \mu$ s were recorded with a Melles Griot wide bandwidth amplifier for the same $1.43 \mathrm{~mm}$ thick LiZnAs device biased at 10 volts, indicating charge carrier induction.

Neutron sensitivity was evaluated with LiZnP and LiZnAs unpurified and purified devices. The first neutron induced signals from Nowotny-Juza materials were observed in this study. Unpurified samples suffered from noise "bursts" that required that measurements be made with constant observation to ensure that noise would not affect the measurement as it was collected. If a noise burst was observed, the measurement was restarted so that a clean measurement would be collected. Purification of the synthesized material was performed to help increase the material quality and reduce the noise bursts observed with the unpurified devices. Devices fabricated from purified material did not suffer from the same noise bursts; however, devices would often breakdown and suffer from continuous noise after prolonged testing with a high applied voltage $(> \pm 10$ volts measured at the detector), a problem that was also observed with unpurified devices. It is suspected that possibly in addition to contact breakdown, that conduction paths were created as a result of increased bias and/or prolonged applied bias to devices. These materials suffered 
from many material problems that all influence the device performance, including charge trapping and charge carrier scattering largely attributed to impurities. Nevertheless, neutron sensitivity was observed, where some devices performed better than others. It is likely that samples 'mined' from certain areas of the ingot were of higher material quality than other areas, thereby, leading to better device performance.

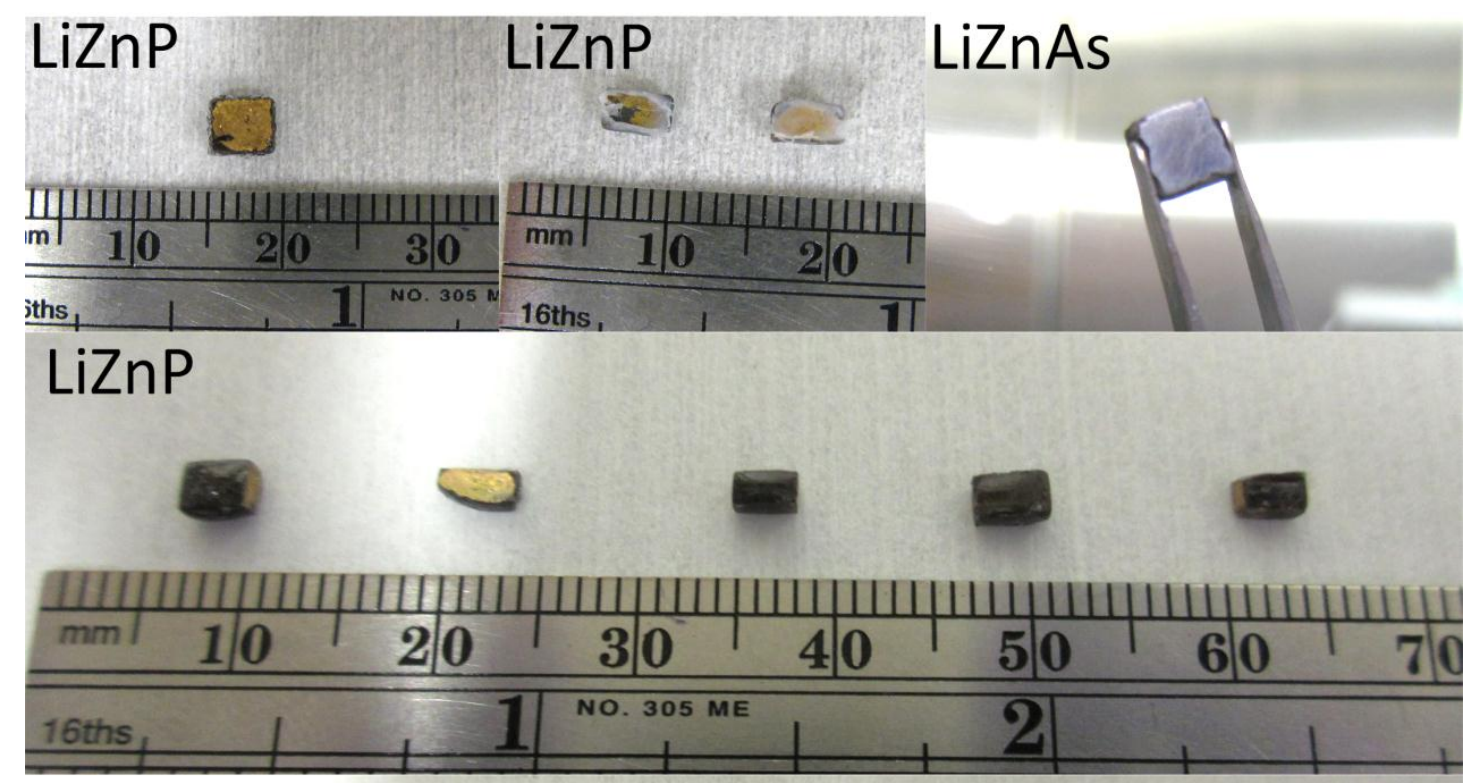

\section{LiZnAs}

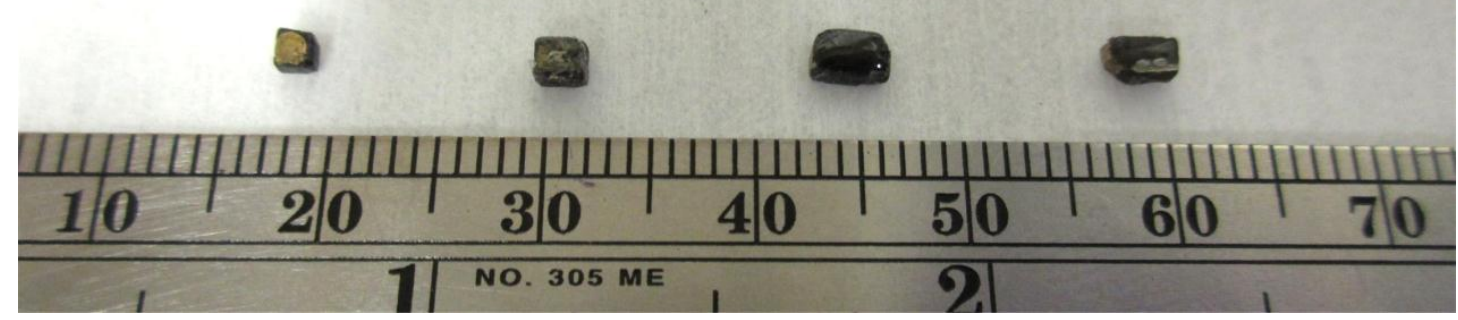

Fig. 1. A number of purified bulk LiZnP and LiZnAs samples that were grown by the hightemperature Bridgman method [4, 5]. The sample on the top left has dimensions of $2.020 \times 3.518$ $\mathrm{x} 4.077 \mathrm{~mm}^{3}$. The two LiZnP samples across the top are coated in HumiSeal ${ }^{\circledR}$. After reprocessing, all samples were coated in Epoxy Tech. 301-2, as shown for all of the samples across the middle and bottom pictures. 


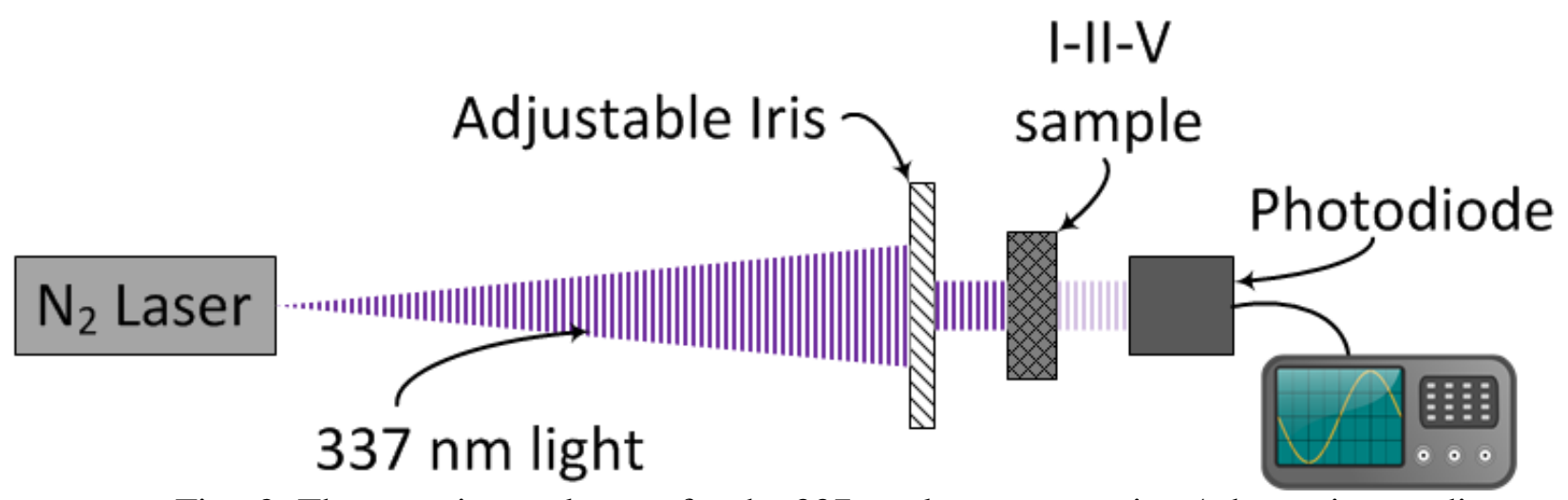

Fig. 2. The experimental setup for the $337 \mathrm{~nm}$ laser attenuation / absorption studies.

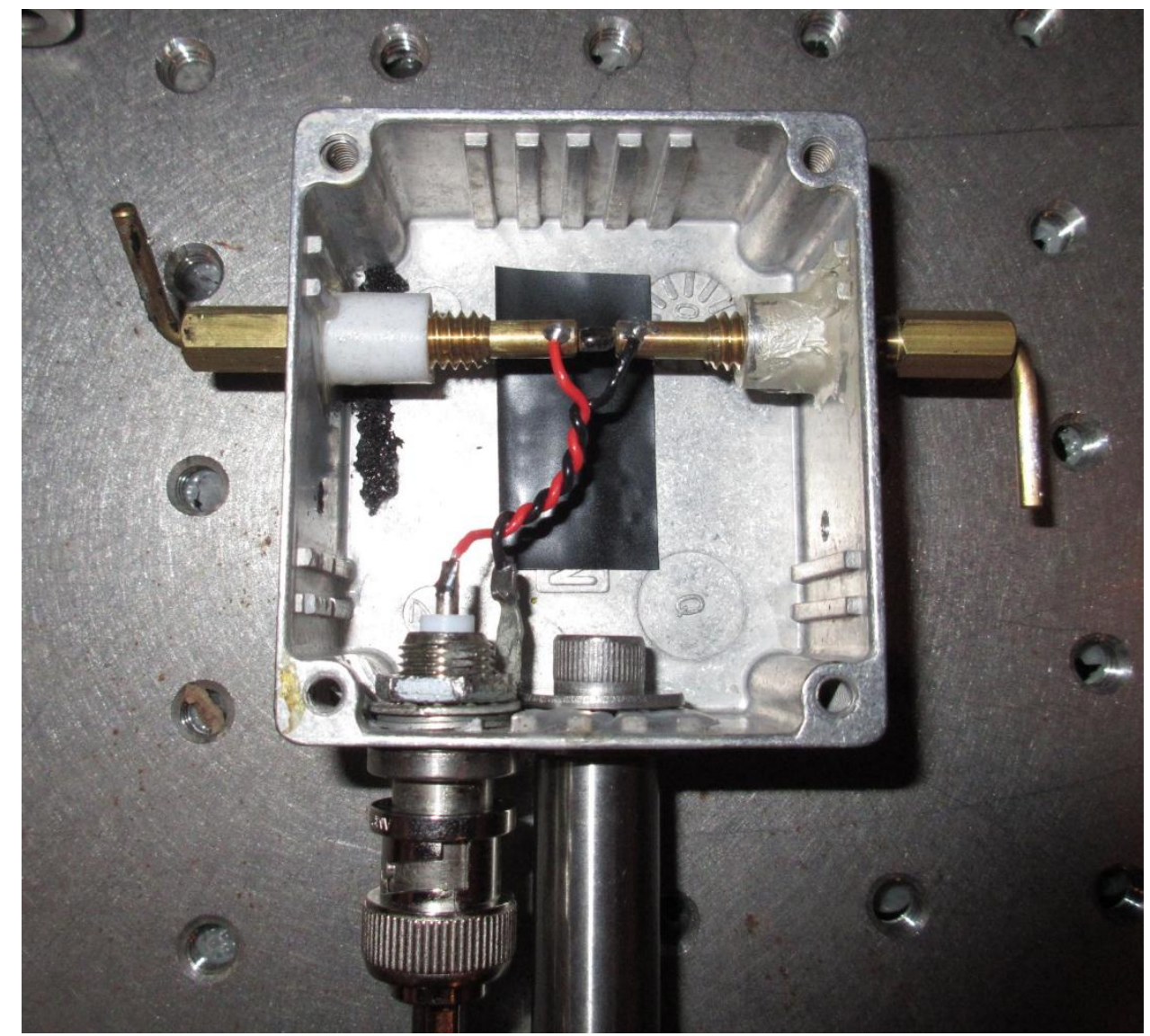

Fig. 3. The project box for testing LiZnP and LiZnAs samples. A LiZnAs sample (1.43 x $3.96 \mathrm{x}$ $2.45 \mathrm{~mm}^{3}$ ) is mounted between the electrodes. 


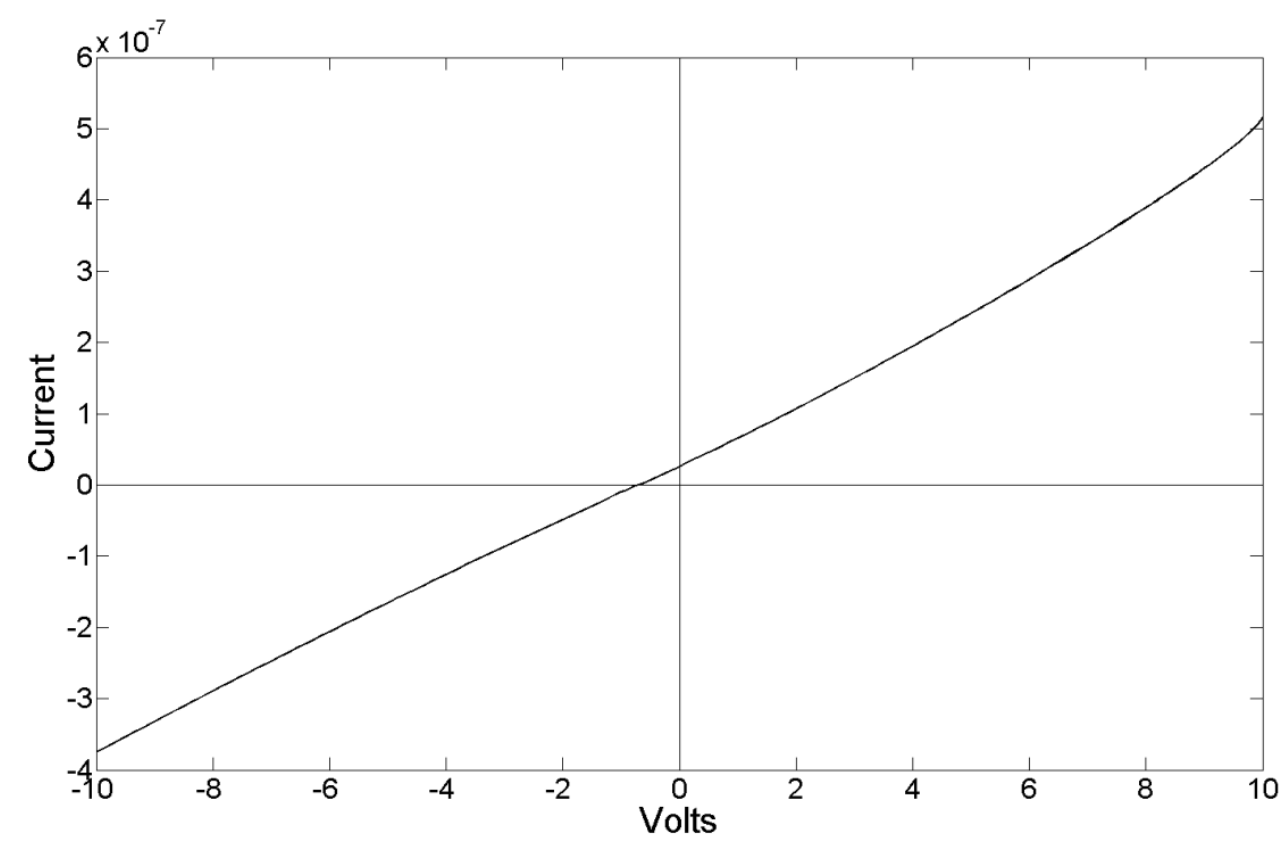

Fig. 4. An I-V response from a purified LiZnAs device $\left(2.100 \times 4.060 \times 4.155 \mathrm{~mm}^{3}\right)$. Resistivity was determined to be $1.6 \times 10^{7} \Omega \mathrm{cm}$.

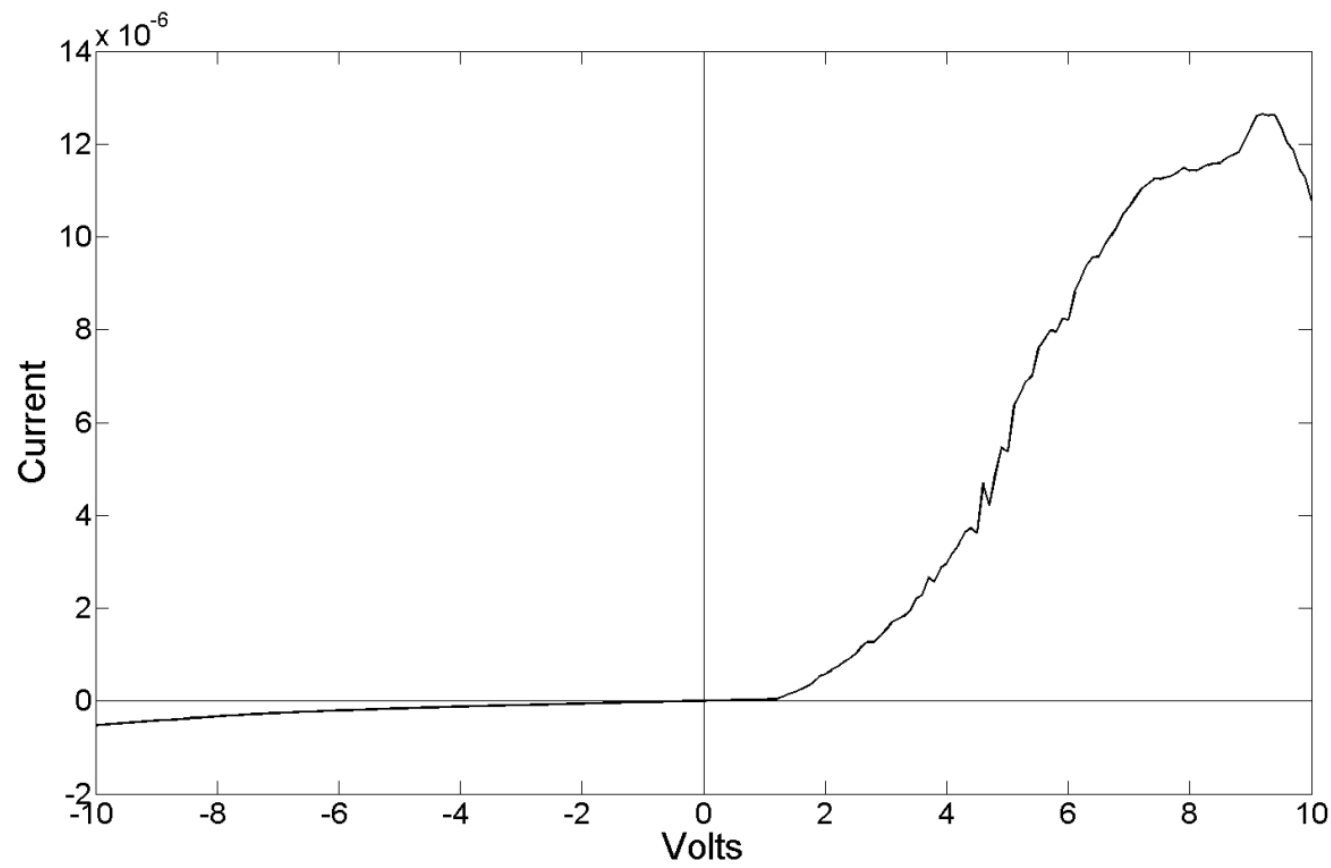

Fig. 5. An I-V response from a purified LiZnAs device of dimensions $1.43 \times 3.96 \times 2.45 \mathrm{~mm}^{3}$. This device exhibited a rectifying I-V characteristic. Resistivity was determined to be $1.7 \times 10^{6}$ $\Omega \mathrm{cm}$ at -10 volts. 


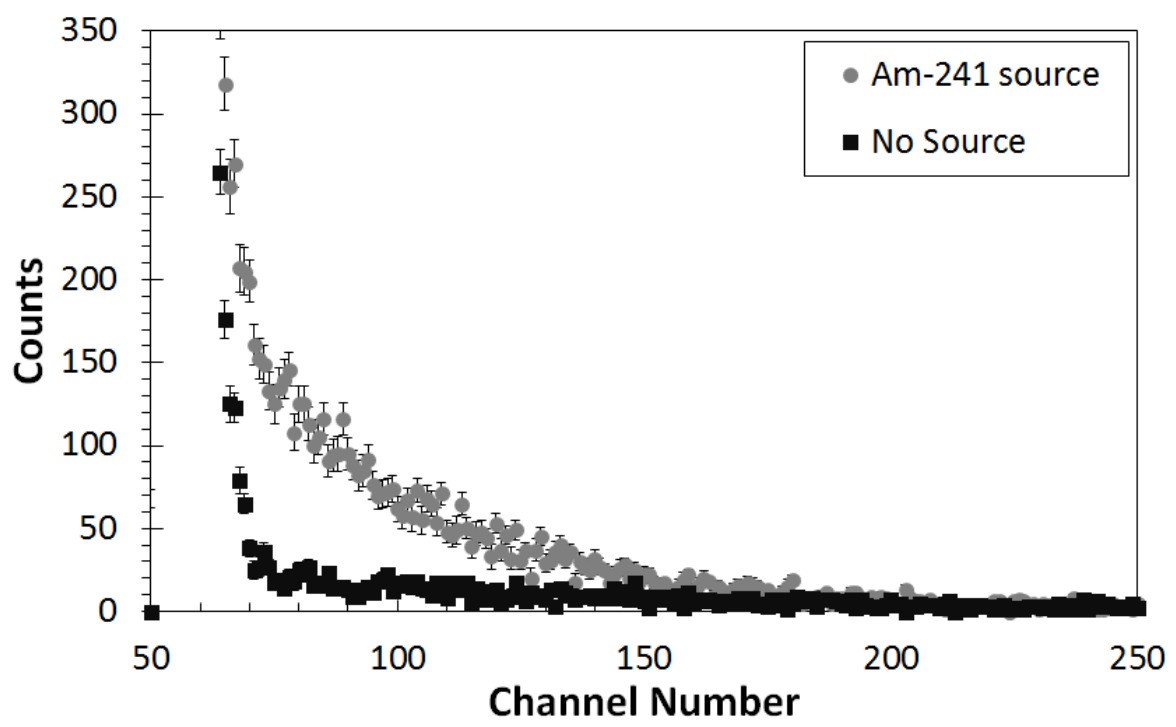

Fig. 6. The pulse height spectrum for a 600 second measurement of the response from $5.48 \mathrm{MeV}$ alpha particles incident the cathode of a LiZnP sample at 50 volt detector bias. The lower level discriminator was set at channel number 60.

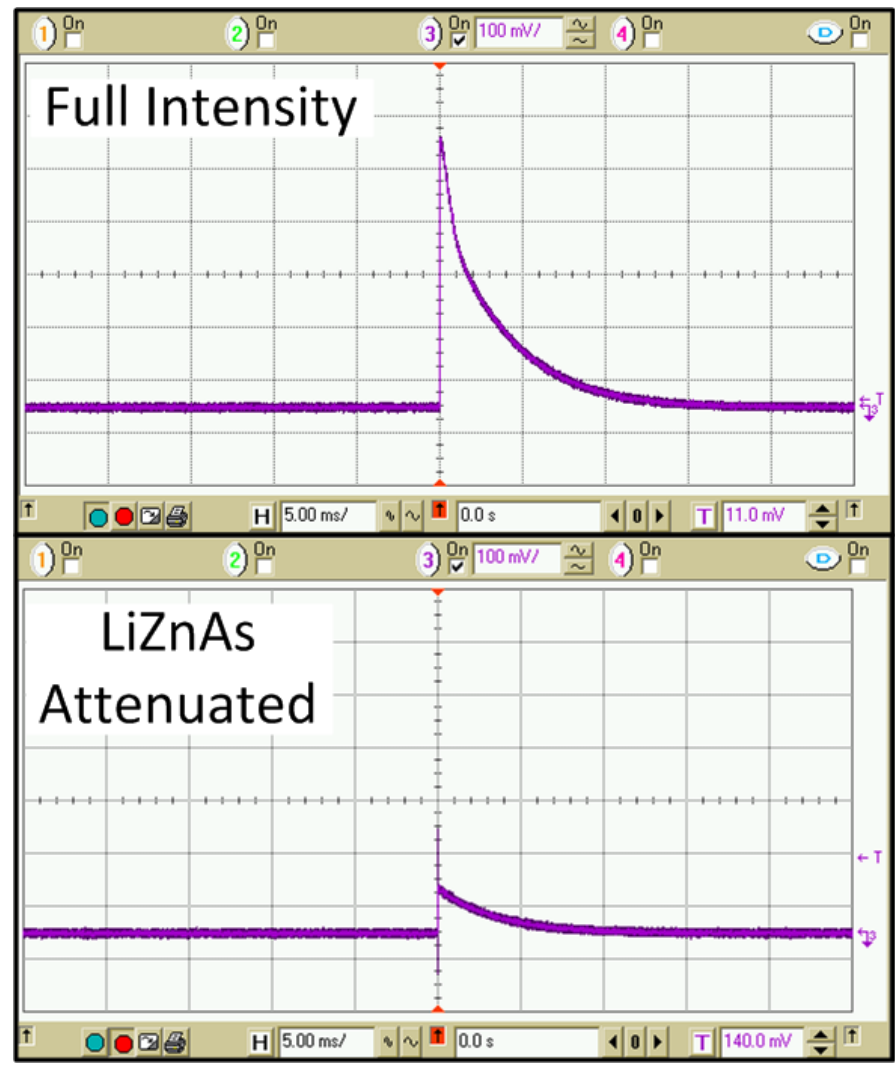

Fig. 7. The response from the Si photodiode witness from a $1.0 \mathrm{~mm}$ collimated $337 \mathrm{~nm}$ laser pulse (top) and the response from the Si photodiode witness with the LiZnAs sample in the $337 \mathrm{~nm}$ collimated beam (bottom). 


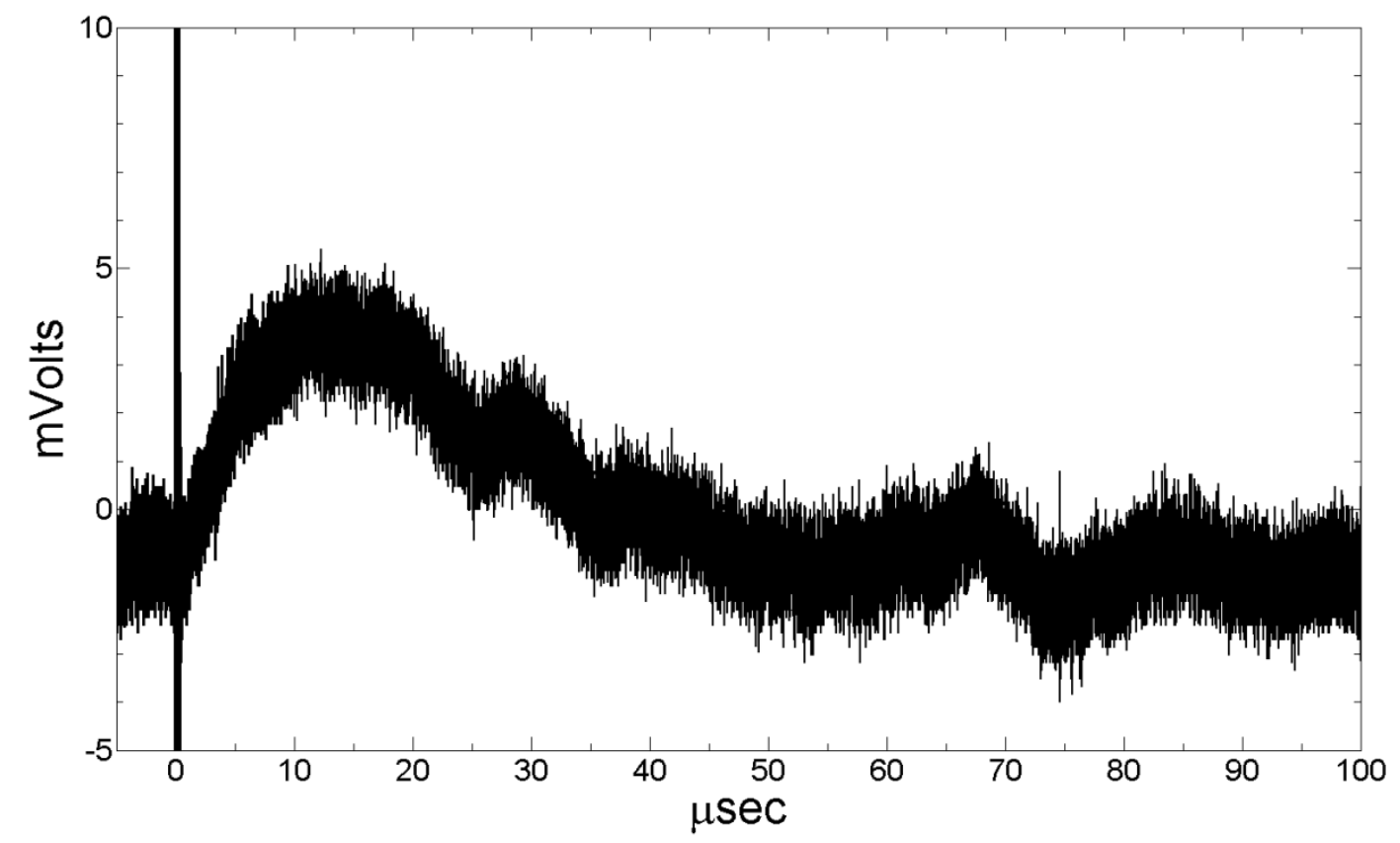

Fig. 8. The resulting waveform from a LiZnAs sample $\left(1.59 \times 1.39 \times 1.42 \mathrm{~mm}^{3}\right)$ connected to the Melles Griot wide bandwidth amplifier and irradiated with a $1.0 \mathrm{~mm}$ collimated $250 \mu \mathrm{J}$ pulse of $337 \mathrm{~nm}$ laser as recorded on the oscilloscope.

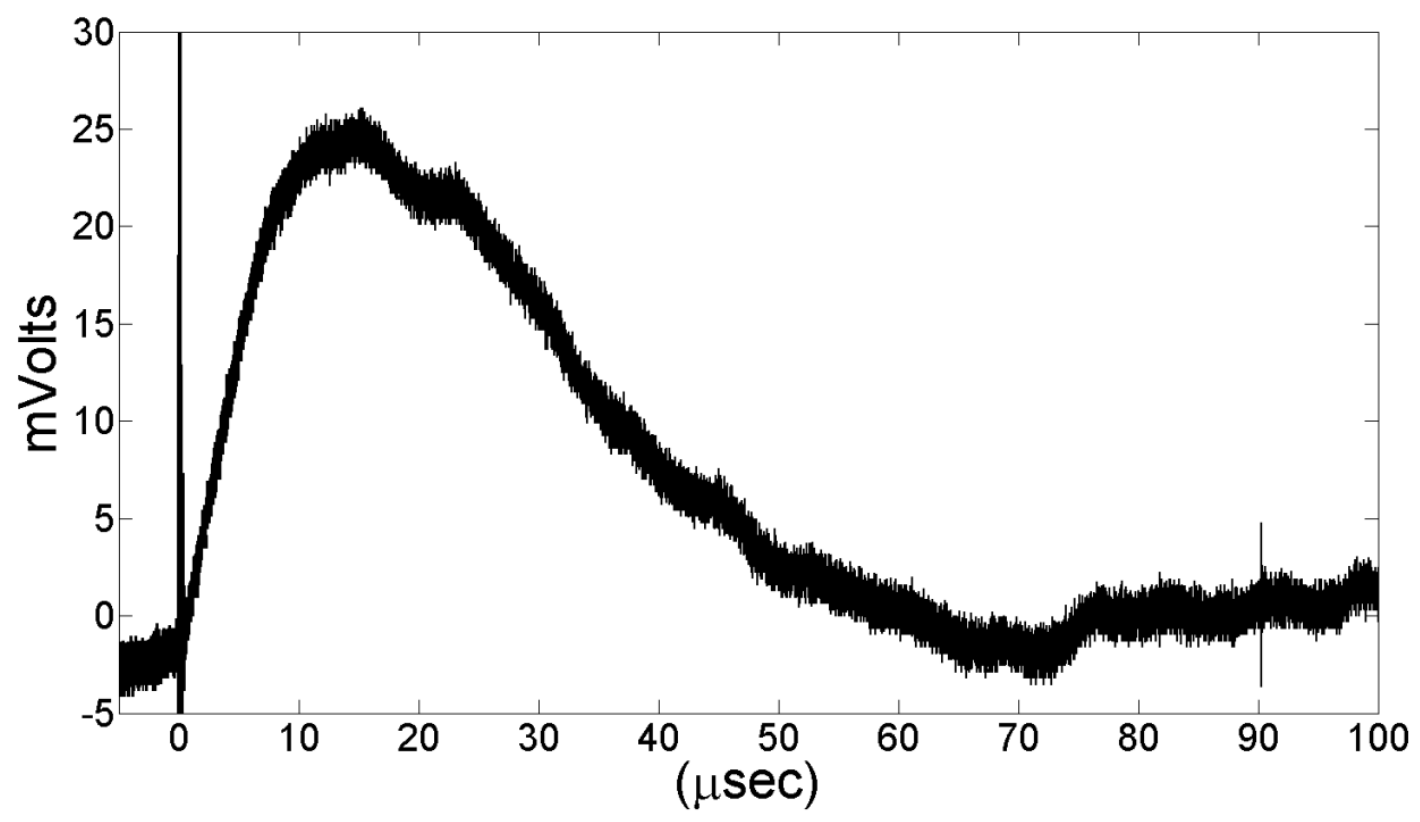

Fig. 9. The resulting waveform from a LiZnAs sample $\left(1.43 \times 3.96 \times 2.45 \mathrm{~mm}^{3}\right)$ connected to the Melles Griot wide bandwidth amplifier and irradiated with a $1.0 \mathrm{~mm}$ collimated $250 \mu \mathrm{J}$ pulse of $337 \mathrm{~nm}$ laser, as recorded on the oscilloscope. 


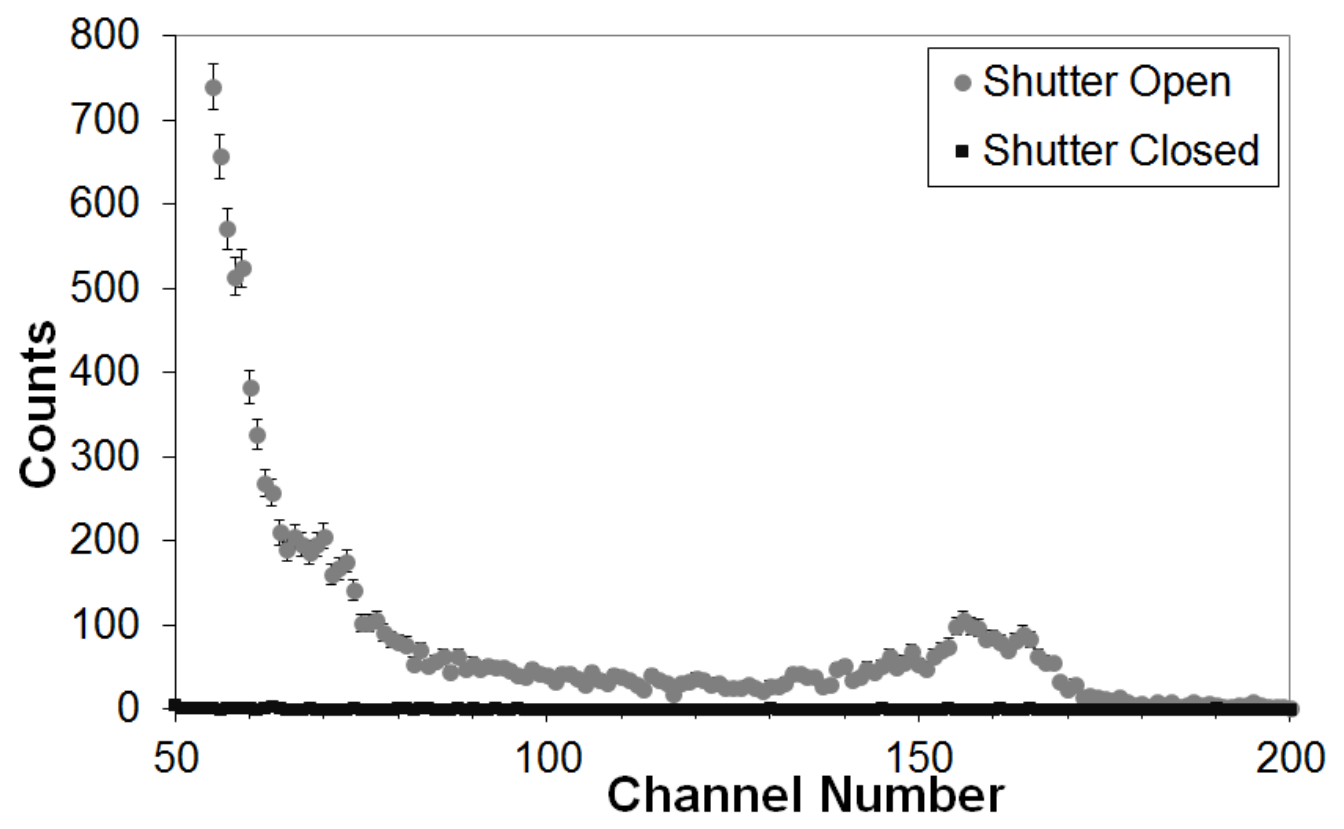

Fig. 10. A thermal neutron response measurement with the LiZnP sample. Shown are 300 second measurements collected at a reactor power of $200 \mathrm{~kW}$ and -10 volts applied to the detector. The LLD was set to channel number 47.

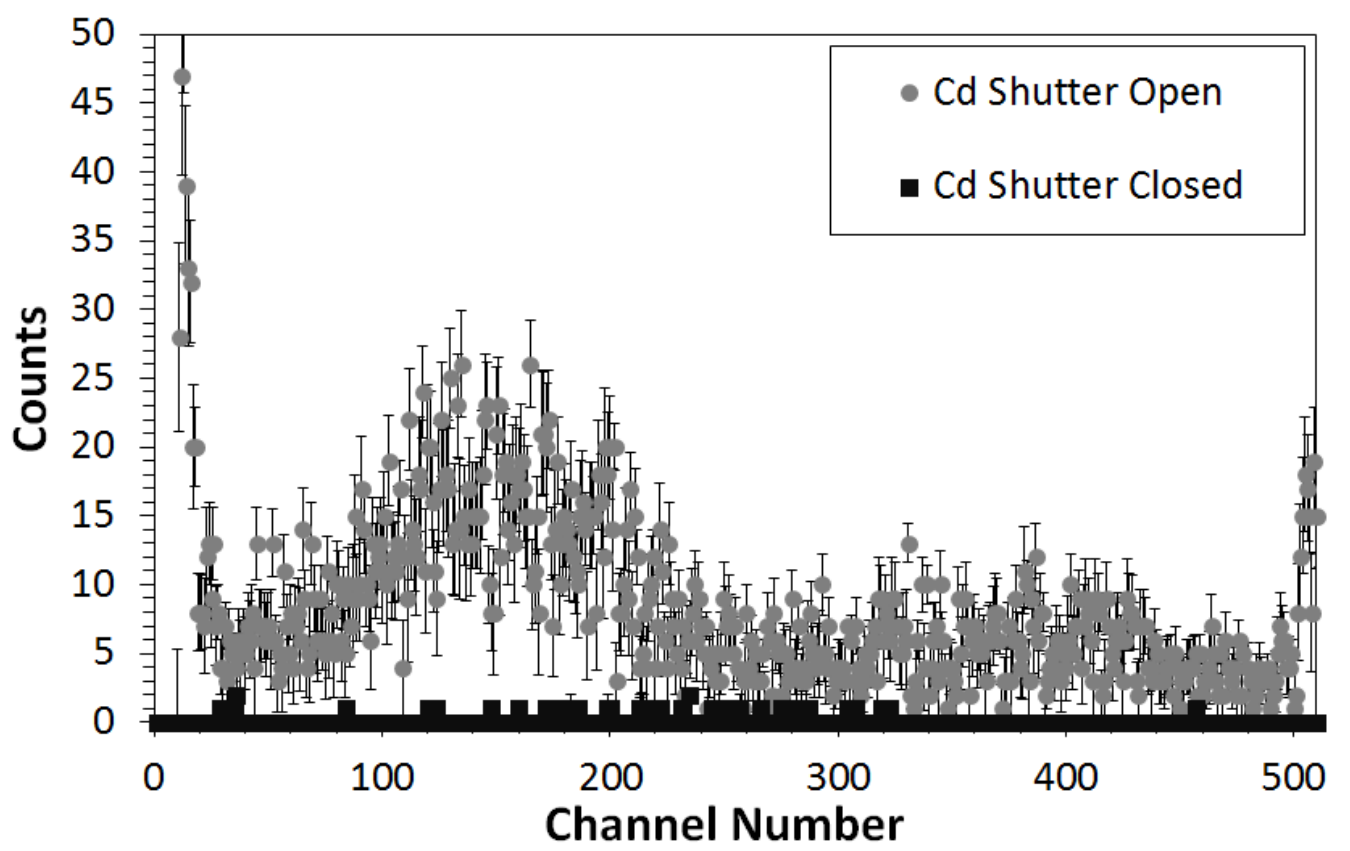

Fig. 11. Thermal neutron reaction product pulse height spectrum with a LiZnP sample of dimensions $2.020 \times 3.518 \times 4.077 \mathrm{~mm}^{3}$ with 46 volts applied to the detector. Measurements were collected at the thermal neutron beam port at a $200 \mathrm{~kW}$ reactor power. $300 \mathrm{sec}$ measurements were collected. 


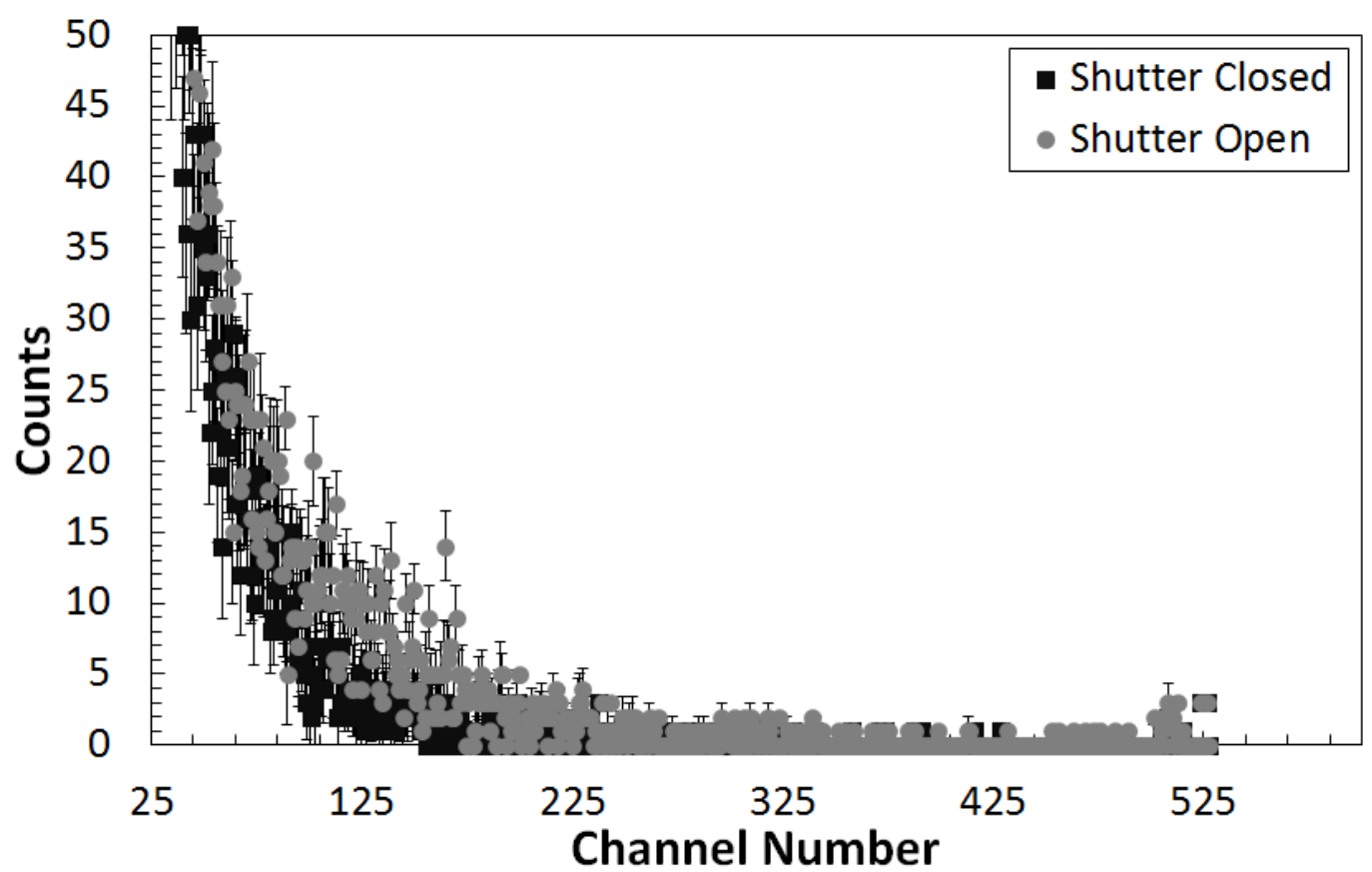

Fig. 12. Thermal neutron response pulse height spectrum with the same LiZnP sample with the response in Fig. $11\left(2.020 \times 3.518 \mathrm{~mm} \times 4.077 \mathrm{~mm}^{3}\right) .48$ volts applied to the detector. Measurements were collected for 300 seconds at $200 \mathrm{~kW}$ reactor power.

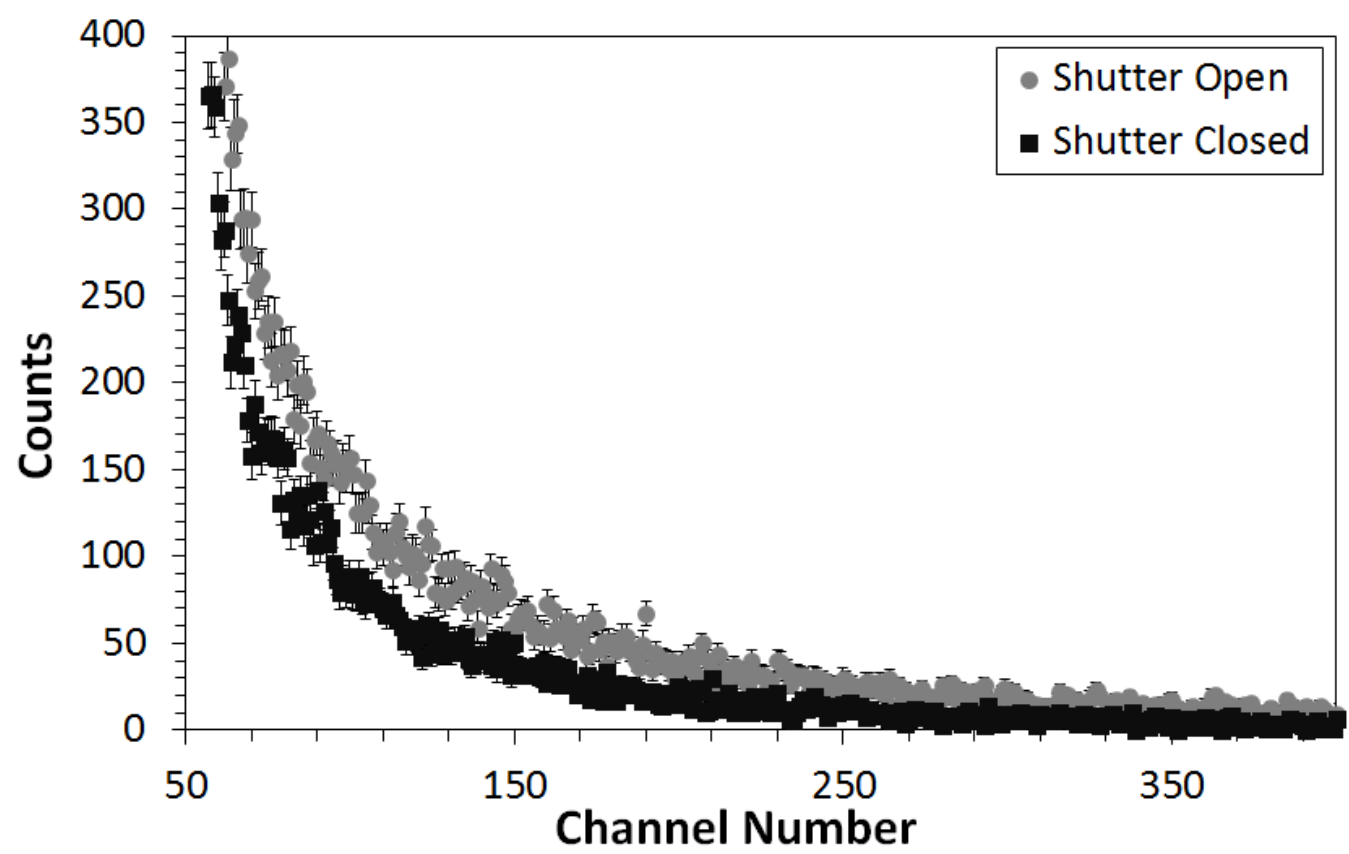

Fig. 13. Thermal neutron reaction product pulse height spectrum with a LiZnAs sample of dimensions $2.100 \times 4.060 \times 4.155 \mathrm{~mm}^{3}$ with 53 volts applied to the detector. Measurements were collected for 600 seconds at a reactor power of $250 \mathrm{~kW}$. 


\section{ACKNOWLEDGEMENTS}

The authors express gratitude to Dr. Brett DePaola, and the Kansas State University Physics department for the use of their Laser Photonics LN300 laser and optical equipment. Work funded in part by the Advanced Materials program DOE - NNSA, grant \# DE-FEG52-08NA28766.

\section{REFERENCES}

[1] B. W. Montag, M. A. Reichenberger, K. R. Arpin et al., "Synthesis and Characterization of LiZnP and LiZnAs Semiconductor Material," J. of Cryst. Growth, vol. 412, pp. 103108, Feb 15, 2015.

[2] B. W. Montag, M. A. Reichenberger, P. B. Ugorowski et al., "Static Sublimation Purification Process and Characterization of LiZnP Semiconductor Material," J. of Cryst. Growth, vol. 419, pp. 133-137, June 1, 2015.

[3] B. W. Montag, M. A. Reichenberger, P. B. Ugorowski et al., "Static Sublimation Purification Process and Characterization of LiZnAs Semiconductor Material," J. of Cryst. Growth, vol. 438, pp. 99-103, 2016, 2016.

[4] B. W. Montag, M. A. Reichenberger, M. Sunder et al., "Bulk crystal growth, and highresolution x-ray diffraction results of LiZnP semiconductor material," Journal of Crystal Growth, vol. 419, pp. 143-148, June 1, 2015.

[5] B. W. Montag, M. A. Reichenber, M. Sunder et al., "Bulk Crystal Growth, and HighResolution X-Ray Diffraction Results of LiZnAs Semiconductor Material," Journal of Electronic Materials, pp., Submitted, 2016.

[6] R. Juza, K. Langer, and K. Von Benda, "Ternary Nitrides, Phosphides, and Arsenides of Lithium," Angew. Chem. Int. Edit., vol. 7, no. 5, pp. 360-370, 1968.

[7] H. Nowotny, and K. Bachmayer, "Die Verbindungen LiMgP, LiZnP und LiZnAs," Monatsh. Chem. und verwandte Teile anderer Wissenschaften, vol. 81, no. 4, pp. 488496, 1950.

[8] D. Kieven, A. Grimm, A. Beleanu et al., "Preparation and Properties of RadioFrequency-Sputtered Half-Heusler Films for Use in Solar Cells," Thin Solid Films, vol. 519, no. 6, pp. 1866-1871, 2011.

[9] Z. W. Bell, K. R. Pohl, and L. Van Den Berg, "Neutron Detection with Mercuric Iodide," IEEE Trans. Nuc. Sci., vol. 51, no. 3, pp. 1163-1165, 2004.

[10] A. G. Vradii, M. I. Krapivin, L. V. Maslova et al., "Possibilities of Recording Thermal Neutrons with Cadmium Telluride Detectors," Sov.Atom. Energy., vol. 42, no. 1, pp. 6466, 1977.

[11] A. G. Beyerle, and K. L. Hull, "Neutron Detection with Mercuric Iodide Detectors," Nucl. Inst. and Meth. A, vol. 256, no. 2, pp. 377-380, 1987.

[12] D. S. McGregor, J. T. Lindsay, and R. W. Olsen, "Thermal Neutron Detection with Cadmium $_{1-\mathrm{x}}$ Zinc $_{\mathrm{x}}$ Telluride Semiconductor Detectors," Nucl. Inst. and Meth. A, vol. 381, no. 2, pp. 498-501, 1996.

[13] Y. Kumashiro, K. Kudo, K. Matsumoto et al., "Thermal Neutron Irradiation Experiments on ${ }^{10} \mathrm{BP}$ Single-Crystal Wafers," J. Less Common Met., vol. 143, no. 1-2, pp. 71-75, 1988. 
[14] W. C. McGinnis, Film Implementation of a Neutron Detector (FIND): Proof of Concept Device, SPAWAR Systems Center, Technical Report 1921, 2003.

[15] J. C. Lund, F. Olschner, F. Ahmed et al., "Boron Phosphide on Silicon for Radiation Detectors," MRS Online P. Libr., vol. 162, pp. 601-604, 1989.

[16] F. P. Doty, Boron Nitride Solid State Neutron Detector, US-6727504, 2004.

[17] A. N. Caruso, P. A. Dowben, S. Balkir et al., "The All Boron Carbide Diode Neutron Detector: Comparison with Theory," Mater. Sci. Eng. B, vol. 135, no. 2, pp. 129-133, 2006.

[18] B. W. Robertson, S. Adenwalla, A. Harken et al., "A Class of Boron-Rich Solid-State Neutron Detectors," Appl. Phys. Lett., vol. 80, no. 19, pp. 3644-3646, 2002.

[19] P. Groot, J. H. F. Grondel, and P. J. v. d. Put, "Chemical Vapour Deposition of Boron Phosphides Using Bromide Etchants," Solid State Ionics, vol. 16, pp. 95-98, 1985.

[20] A. N. Caruso, R. B. Billa, S. Balaz et al., "The Heteroisomeric Diode," J. Phys. Condens. Matter, vol. 16, pp. L139-L146, 2004.

[21] D. S. McGregor, M. D. Hammig, Y. H. Yang et al., "Design Considerations for Thin Film Coated Semiconductor Thermal Neutron Detectors-I: Basics Regarding Alpha Particle Emitting Neutron Reactive Films," Nucl. Inst. and Meth. A, vol. 500, no. 1-3, pp. 272-308, 2003.

[22] K. S. Shah, "Solid State Neutron Detection with LiZnP," DOE.

[23] K. Geels, Metallographic and Materialographic Specimen Preparation, Light Microscopy, Image Analysis and Hardness Testing, West Conshohocken, PA 194282959: ASTM International, 2007.

[24] Pace Technologies Inc., "Abrasive Grinding Paper," http://www.metallographic.com/Brochures/SiCpaper.pdf, 2012.

[25] K. Kuriyama, T. Kato, and K. Kawada, "Optical Band Gap of the Filled Tetrahedral Semiconductor LiZnAs," Phys. Rev. B: Condens. Matter, vol. 49, no. 16, pp. 11452-5, 1994.

[26] K. Kuriyama, T. Katoh, and N. Mineo, "Crystal Growth and Characterization of the Filled Tetrahedral Semiconductor Lithium Zinc Phosphide (LiZnP)," J. Cryst. Growth, vol. 108, no. 1-2, pp. 37-40, 1991.

[27] G. Lutz, Semiconductor Radiation Detectors, New York, NY: Springer Science, 1999.

[28] J. F. Ziegler, "SRIM / TRIM: The Stopping and Range of Ions in Matter," 2013. 